\section{Acinetobacter lwoffii Septicemia Associated with a Peripheral Intravascular Catheter}

Takehiko Mori, Tomonori Nakazato, Rie Yamazaki, Yasuo Ikeda and Shinichiro Okamoto

Key words: Acinetobacter lwoffii, septicemia, intravascular catheter

\section{(DOI: 10.2169/internalmedicine.45.1580)}

Acinetobacter is an aerobic, non-fermentative gramnegative bacillus, which is widespread in nature (1). Acinetobacter has been recognized as a pathogen which plays a significant role in the colonization and infection in immunocompromised patients. Acinetobacter has been implicated in a variety of nosocomial infections, including bacteremia, urinary tract infection, and ventilator-associated pneumonia, further complicating the illness with its increasing incidence of multi-drug resistance $(1,2)$. The species other than Acinetobacter baumannii, including Acinetobacter lwoffii, are less frequently isolated and used to be considered as contaminants derived from the environment. However, a limited number of cases of infection caused by Acinetobacter lwoffii has been reported, most of which are central intravascular catheter-related blood stream infections or bacteremia (3-7). Here, we report a case of septicemia due to Acinetobacter lwoffii shortly after removal of a peripheral intravascular catheter.

A 43-year-old woman with myelodysplastic syndrome underwent allogeneic bone marrow transplantation from her human leukocyte antigen-matched sister. Her early posttransplant course during neutropeina was remarkable with bacteremia due to Staphylococcus epidermidis, which was successfully treated with teicoplanin. After leukocyte recovery was obtained, she developed mild acute graft-versus-host disease (GVHD), which was treated with cyclosporin A (CSA) and prednisolone. Prednisolone was tapered off on day 84 post-transplant. On day 120 post-transplant, when she was still treated with CSA, she developed a seizure, which was diagnosed as CSA-associated encephalopathy. She completely recovered after CSA administration was discontinued. On day 134 post-transplant, when she was afebrile and had a normal white blood cell count $(3,700 / \mu 1$, in- cluding $72 \%$ of neutrophils), she complained of pain and swelling at the site of the peripheral intravascular catheter on her right forearm. Immediately after removing the catheter, she developed chilling, high-grade fever, and hypotension (blood pressure 68 over $24 \mathrm{mmHg}$ ), accompanied by oliguria and marked elevation of c-reactive protein (12.9 mg/dl). Blood culture yielded Acinetobacter lwoffii, which was susceptible to piperacillin, ceftazidime, imipenem, and gentamicin. Based on these findings, septicemia, catheterrelated bacteremia with systemic inflammatory response syndrome, due to Acinetobacter lwoffii was diagnosed. She was treated with intravenous administration of piperacillin and gentamicin, and continuous infusion of dopamine and norepinephrine to maintain the blood pressure, which successfully improved her condition. After initiating this combination antimicrobial chemotherapy, repeated blood cultures failed to detect the bacillus. In 7 days after the onset, she completely recovered without any abnormal findings both in physical examination and laboratory data. No other cases of Acinetobacter lwoffii infection were observed around this period.

Acinetobacter lwoffii infrequently causes infections in humans, and there have been only a few reports describing catheter-related bloodstream infections or bacteremia due to Acinetobacter lwoffii (4-7). Most of these reported cases of Acinetobacter lwoffii bacteremia are associated with central intravascular catheter, which had a good prognosis with the prompt removal of the catheter and initiation of antimicrobial chemotherapy. In our case, a peripheral intravascular catheter was considered the portal of Acinetobacter lwoffii, since the pain and swelling of its inserted site preceded the onset of septicemia and no other focus of infection was found. Furthermore, it is notable that septicemia occurred immediately after removing the catheter, which strongly suggested that removal of catheter triggered the entry of bacillus into the blood stream.

In conclusion, physicians should recognize Acinetobacter lwoffii as one of the pathogens causing sepeticemia, which is not only associated with a central intravascular catheter but also a peripheral intravascular catheter. In addition, although the removal of an involved catheter is recommended in catheter-related infections, it should be noted that its removal may trigger the development of septicemia from local infection. 


\section{References}

1. Bergogne-Berezin E, Towner KJ. Acinetobacter spp. as nosocomial pathogens: microbiological, clinical, and epidemiological features. Clin Microbiol Rev 9: 148-165, 1996.

2. Forster DH, Daschner FD. Acinetobacter species as nosocomial pathogens. Eur J Clin Microbiol Infect Dis 17: 73-77, 1998.

3. Galvao C, Swartz R, Rocher L, Reynolds J, Starmann B, Wilson D. Acinetobacter peritonitis during chronic peritoneal dialysis. Am J Kidney Dis 14: 101-104, 1989.

4. Rolston K, Guan Z, Bodey PG, Elting L. Acinetobacter calcoaceticus septicemia in patients with cancer. South Med J 78: 647-651, 1985.
5. Seifert H, Strate A, Schulze A, Pulverer G. Vascular catheterrelated bloodstream infection due to Acinetobacter johnsonii (formerly Acinetobacter calcoaceticus var. lwoffii); report of 13 cases. Clin Infect Dis 17: 632-636, 1993.

6. $\mathrm{Ku}$ SC, Hsueh PR, Yang PC, Luh KT. Clinical and microbiological characteristics of bacteremia caused by Acinetobacter lwoffii. Eur J Clin Microbiol Infect Dis 19: 501-505, 2000.

7. Fuchs GJ, Jaffe N, Pickering LK. Acinetobacter calcoaceticus sepsis in children with malignancies. Pediatr Infect Dis 5: 545-549, 1986.

(C) 2006 The Japanese Society of Internal Medicine

http://www.naika.or.jp/imindex.html 